\title{
New Friedelane Triterpenoids with Antimicrobial Activity from the Stems of Drypetes paxii
}

\author{
David Dako Chiozem, ${ }^{a}$ Hanh Trinh-Van-Dufat, ${ }^{b}$ Jean Duplex Wansi ${ }^{c}$, Céline Mbazoa Duama, ${ }^{a}$ \\ Véronique Simone Fannang, ${ }^{a}$ Elisabeth Seguin, ${ }^{d}$ François Tillequin, ${ }^{b}$ and Jean WANDJI ${ }^{*, a}$ \\ ${ }^{a}$ Laboratoire de Synthèse et de Substances Naturelles, Département de Chimie Organique de l'Université de Yaoundé-1, \\ Faculté des Sciences; P. O. Box 812 Yaoundé, Cameroun: ${ }^{b}$ Laboratoire de Pharmacognosie de l'Université Paris \\ Descartes, UMR/CNRS $N^{\circ}$ 8638, Faculté des Sciences Biologiques et Pharmaceutiques; 4-Avenue de l'Observatoire, \\ 75006 Paris, France: ' Department of Chemistry, University of Douala, Faculty of Science; P. O. Box 24157 Douala, \\ Cameroon: and ${ }^{d}$ Laboratoire de Pharmacognosie, UFR de Médecine Pharmacie Rouen; 22-Bld Gambetta, 76183 Rouen \\ Cedex 1, France. $\quad$ Received April 7, 2009; accepted June 19, 2009; published online July 2, 2009
}

Two new friedelane-type triterpenes named $12 \alpha$-hydroxyfriedelane-3,15-dione and $3 \beta$-hydroxyfriedelan-25al, together with six known compounds were isolated from the stems of Drypetes paxii Hutch. (Euphorbiaceae). Their structures were established on the basis of conventional 1 dimensional (1D) NMR methods, 2D shift-correlated NMR experiments and mass spectra. The five friedelane-type triterpene derivatives and one olean-12-ene triterpene saponin were tested for antimicrobial activity against some Gram-positive and Gram-negative bacteria, and they appeared to be modestly active.

Key words Drypetes paxii; Euphorbiaceae; stem; friedelane-type triterpenoid; antimicrobial activity

The genus Drypetes belongs to the family Euphorbiaceae and is widely used in West and Central Africa for diverse therapeutic applications such as, the treatment of sinusitis, swellings, boils, gonorrhoea and dysentery. ${ }^{1-4)}$ Our previous study of some Drypetes species resulted to bioactive compounds including the anti-inflammatory, the analgesic, ${ }^{5,6}$ the antileishmanial ${ }^{7)}$ and the antimicrobial activities. ${ }^{8)}$ As a continuation of our investigations on bioactive compounds from the Drypetes species, we studied the methanol extract of the whole stems of Drypetes paxii, a forest shrub growing in the Centre and East provinces of Cameroon. We isolated two new friedelane-type triterpene derivatives, along with six known compounds identified as, friedelin (3), ${ }^{9)}$ friedelan-7one (4), ${ }^{9)}$ friedelane-3,15-dione (5), ${ }^{9)} \beta$-sitosterol (6), ${ }^{10)} 3 \beta$ hydroxyolean-12-en-28- $\beta$-D-glucopyranosyl ester $(7)^{10)}$ and 3- $O$ - $\beta$-D-glucopyranosyl- $\beta$-sitosterol (8). ${ }^{10)}$ The structures of the new compounds, on the basis of spectroscopic analysis, have been determined as $12 \alpha$-hydroxyfriedelane-3,15-dione (1) and 3 $\beta$-hydroxyfriedelan-25-al (2). In the present paper, their isolation, structural determination and the antimicrobial activity of compounds $\mathbf{1}-\mathbf{5}$ and $\mathbf{7}$ will be described.

\section{Results and Discussion}

The methanol extract of the air dried stems of Drypetes paxii was chromatographed on a column of silica gel eluted with hexane, EtOAc and $\mathrm{MeOH}$ in increasing polarity to afford compounds $\mathbf{1}-\mathbf{8}$. Compounds $\mathbf{3}-\mathbf{8}$ were identified as known compounds on comparison of their spectral data with those reported in the literature.

Compound 1, colourless amorphous crystals; mp 199$201{ }^{\circ} \mathrm{C}$, gave positive reaction to the Libermann-Burchard test. Its molecular formula was determined to be $\mathrm{C}_{30} \mathrm{H}_{48} \mathrm{O}_{3}$ based on the high resolution time of flight electro spray mass spectrometry (HR TOF ES ${ }^{+} \mathrm{MS}$ ) $m / z$ 456.7152, the $\mathrm{CI} / \mathrm{NH}_{3}$ MS $m / z 457[\mathrm{M}+\mathrm{H}]^{+}, 474\left[\mathrm{M}+\mathrm{NH}_{4}\right]^{+}$, the EI-MS $m / z 456$ $[\mathrm{M}]^{+}$and the ${ }^{13} \mathrm{C}-\mathrm{NMR}$ spectrum which exhibited 30 carbon signals. The IR spectrum displayed vibration bands at 3410 $(\mathrm{OH})$ and $1725(\mathrm{C}=\mathrm{O}) \mathrm{cm}^{-1}$. The ${ }^{1} \mathrm{H}$ - and ${ }^{13} \mathrm{C}-\mathrm{NMR}$ spectra
(Table 1$)$ showed one oxymethine signals $\left(\delta_{\mathrm{C}} 74.1\right.$ and $\delta_{\mathrm{H}}$ 3.97) and two ketone resonances at $\delta_{\mathrm{C}} 213.1$ and 212.1. In addition, the ${ }^{1} \mathrm{H}$-detected heteronuclear single quantum coherence (HSQC) and the distortionless enhancement by polarization transfer (DEPT) spectra displayed signals for eight methyls, nine methylenes and five methines (one of which is oxygenated). The carbon signal at $\delta_{\mathrm{C}} 6.9$ (C-23) was characteristic for a friedelane-type triterpene skeleton with one of the ketone groups located at the C-3 position. ${ }^{9)}$ The relative positions of the second ketone and the hydroxy group were determined on the basis of the ${ }^{1} \mathrm{H}-{ }^{1} \mathrm{H}$ shift correlation spectroscopy $\left({ }^{1} \mathrm{H}-{ }^{1} \mathrm{H}\right.$ COSY $)$, the ${ }^{1} \mathrm{H}$-detected heteronuclear multiple-bond connectivity (HMBC), the nuclear Overhauser effect spectroscopy (NOESY) correlation and some key ion fragments from the EI-MS. The HMBC spectrum showed long range correlations from proton signal at $\delta_{\mathrm{H}} 3.97$ to carbon signals at $\delta_{\mathrm{C}} 48.7$ (C-9), 56.0 (C-14) and 53.9 (C-18). Therefore the hydroxy group was deduced to be located at the $\mathrm{C}-12$ position. It also exhibited correlations from proton signal at $\delta_{\mathrm{H}} 1.78(\mathrm{H}-8)$ to carbon signals at $\delta_{\mathrm{C}} 59.3(\mathrm{C}-10)$, 29.7 (C-11), 43.0 (C-13) and 212.1, allowing the location of the second ketone at the $\mathrm{C}-15$ position. The correlations from the methyl-26 proton singlet at $\delta_{\mathrm{H}} 1.32$ to carbon signal at $\delta_{\mathrm{C}}$ 212.1 also confirmed the 15 -ketone. The ion fragments at $\mathrm{m} / \mathrm{z}$ 262, 219 and 192 also supported the locations of both hydroxy and ketone groups on rings $\mathrm{C}$ and $\mathrm{D}$ respectively. Furthermore, the NOESY spectrum of compound 1 (Fig. 2) showed cross-peaks from proton signal at $\delta_{\mathrm{H}} 3.97(\mathrm{H}-12)$ to proton signals at $\delta_{\mathrm{H}} 1.32\left(\mathrm{CH}_{3}-26\right), 0.91\left(\mathrm{CH}_{3}-25\right)$ and 2.42 (H-18). Consequently the orientation of the 12-hydroxy group was deduced to be $\alpha$-equatorial. Accordingly, from the above spectroscopic data, the structure of compound $\mathbf{1}$ was assigned as, $12 \alpha$-hydroxyfriedelane-3,15-dione (Fig. 1).

Compound 2 was obtained as a colourless amorphous powder. The GC-MS $m / z 442$ and the $\mathrm{CI} / \mathrm{NH}_{3} \mathrm{MS}, m / z 443$ $[\mathrm{M}+\mathrm{H}]^{+}, 460\left[\mathrm{M}+\mathrm{NH}_{4}\right]^{+}$were consistent with the molecular formula $\mathrm{C}_{30} \mathrm{H}_{50} \mathrm{O}_{2}$ which was confirmed by the HR TOF $\mathrm{ES}^{+} \mathrm{MS} \mathrm{m} / \mathrm{z}$ 442.3825. The ${ }^{13} \mathrm{C}-\mathrm{NMR}$ (Table 1), ${ }^{1} \mathrm{H}-\mathrm{NMR}$, 
Table 1. The ${ }^{1} \mathrm{H}$ - and ${ }^{13} \mathrm{C}-\mathrm{NMR}\left(400,100 \mathrm{MHz}, \mathrm{CDCl}_{3}\right)$ Data $\left.{ }^{a}\right)$ of Compounds $\mathbf{1}$ and 2

\begin{tabular}{|c|c|c|c|c|c|c|}
\hline \multirow{2}{*}{ Atom } & \multicolumn{3}{|c|}{1} & \multicolumn{3}{|r|}{2} \\
\hline & $\delta_{\mathrm{C}}$ & & $\delta_{\mathrm{H}}[\mathrm{m}, J(\mathrm{~Hz})]$ & $\delta_{\mathrm{C}}$ & & $\delta_{\mathrm{H}}[\mathrm{m}, J(\mathrm{~Hz})]$ \\
\hline \multirow[t]{2}{*}{1} & 22.4 & $\mathrm{CH}_{2}$ & $1.97(\mathrm{~m})$ & 17.4 & $\mathrm{CH}_{2}$ & $1.70\left(\mathrm{dt}, 13.0,3.0, \mathrm{H}_{\mathrm{ax}}\right)$ \\
\hline & & & $1.65(\mathrm{~m})$ & & & $1.46\left(-,{ }^{b)} \mathrm{H}_{\mathrm{eq}}\right)$ \\
\hline \multirow[t]{2}{*}{2} & 41.1 & $\mathrm{CH}_{2}$ & $2.35(\mathrm{~m})$ & 36.1 & $\mathrm{CH}_{2}$ & $1.98\left(\mathrm{qd}, 13.0,3.0, \mathrm{H}_{\mathrm{eq}}\right)$ \\
\hline & & & $2.19(\mathrm{~m})$ & & & $1.57\left(-,{ }^{b)} \mathrm{H}_{\mathrm{ax}}\right)$ \\
\hline 3 & 213.1 & $\mathrm{C}$ & & 71.7 & $\mathrm{CH}$ & $3.80\left(\mathrm{q}\right.$-like, $\left.2.0, \mathrm{H}_{\mathrm{eq}}\right)$ \\
\hline 4 & 58.0 & $\mathrm{CH}$ & $2.30(\mathrm{t}, 1.8)$ & 49.5 & $\mathrm{CH}$ & $1.24\left(-,{ }^{b)} \mathrm{H}_{\mathrm{ax}}\right)$ \\
\hline 5 & 41.8 & $\mathrm{C}$ & & 38.3 & $\mathrm{C}$ & \\
\hline \multirow[t]{2}{*}{6} & 39.1 & $\mathrm{CH}_{2}$ & $1.70(\mathrm{~m})$ & 42.3 & $\mathrm{CH}_{2}$ & $1.75\left(\mathrm{td}, 12.0,3.0, \mathrm{H}_{\mathrm{eq}}\right)$ \\
\hline & & & $1.31(-b)$ & & & $0.98\left(-,{ }^{b)} \mathrm{H}_{\mathrm{ax}}\right)$ \\
\hline \multirow[t]{2}{*}{7} & 19.7 & $\mathrm{CH}_{2}$ & $1.57(\mathrm{~m})$ & 17.8 & $\mathrm{CH}_{2}$ & $1.40\left(-^{b)}\right)$ \\
\hline & & & $1.32(\mathrm{~m})$ & & & $1.38\left(-ـ^{b}\right)$ \\
\hline 8 & 35.9 & $\mathrm{CH}$ & 1.78 (br s) & 52.5 & $\mathrm{CH}$ & $1.28\left(-^{b)}\right)$ \\
\hline 9 & 48.7 & $\mathrm{C}$ & & 51.6 & $\mathrm{C}$ & \\
\hline 10 & 59.3 & $\mathrm{CH}$ & $1.43(\mathrm{t}, 7.5)$ & 59.6 & $\mathrm{CH}$ & $0.97\left(\mathrm{dd}, 12.0,2.0, \mathrm{H}_{\mathrm{ax}}\right)$ \\
\hline \multirow[t]{2}{*}{11} & 29.7 & $\mathrm{CH}_{2}$ & $2.25\left(\mathrm{dd}, 10.4,7.7, \mathrm{H}_{\mathrm{ax}}\right)$ & 28.3 & $\mathrm{CH}_{2}$ & $1.45\left(-^{b}\right)$ \\
\hline & & & $1.40\left(\mathrm{dd}, 10.4,3.5, \mathrm{H}_{\mathrm{eq}}\right)$ & & & $1.25\left(-^{b}\right)$ \\
\hline \multirow[t]{2}{*}{12} & 74.1 & $\mathrm{CH}$ & $3.97\left(\mathrm{dd}, 7.7,3.5, \mathrm{H}_{\mathrm{ax}}\right)$ & 31.2 & $\mathrm{CH}_{2}$ & $1.34\left(-^{b)}\right)$ \\
\hline & & & & & & $1.30\left(-^{b)}\right)$ \\
\hline 13 & 43.0 & $\mathrm{C}$ & & 39.2 & $\mathrm{C}$ & \\
\hline 14 & 56.0 & $\mathrm{C}$ & & 38.2 & $\mathrm{C}$ & \\
\hline \multirow[t]{2}{*}{15} & 212.1 & $\mathrm{C}$ & & 31.4 & $\mathrm{CH}_{2}$ & $\left.1.54(-, b) \mathrm{H}-15_{\mathrm{ax}}\right)$ \\
\hline & & & & & & $\left.1.29(-, b) \mathrm{H}-15_{\mathrm{eq}}\right)$ \\
\hline \multirow[t]{2}{*}{16} & 51.4 & $\mathrm{CH}_{2}$ & $2.62\left(\mathrm{~d}, 11.5, \mathrm{H}_{\mathrm{eq}}\right)$ & 35.5 & $\mathrm{CH}_{2}$ & $1.48\left(-^{b)}\right)$ \\
\hline & & & $2.10\left(\mathrm{~d}, 11.5, \mathrm{H}_{\mathrm{ax}}\right)$ & & & $1.20\left(-^{b)}\right)$ \\
\hline 17 & 29.7 & $\mathrm{C}$ & & 30.0 & $\mathrm{C}$ & \\
\hline 18 & 53.9 & $\mathrm{CH}$ & $2.42(\mathrm{~m})$ & 42.8 & $\mathrm{CH}$ & $1.57\left(\mathrm{dd}, 11.0,6.1, \mathrm{H}_{\mathrm{ax}}\right)$ \\
\hline \multirow[t]{2}{*}{19} & 36.0 & $\mathrm{CH}_{2}$ & $1.39(\mathrm{~m})$ & 35.4 & $\mathrm{CH}_{2}$ & $1.38\left(-^{b}\right)$ \\
\hline & & & $1.20(\mathrm{~m})$ & & & $1.22\left(-^{b)}\right)$ \\
\hline 20 & 28.4 & $\mathrm{C}$ & & 28.1 & $\mathrm{C}$ & \\
\hline \multirow[t]{2}{*}{21} & 32.1 & $\mathrm{CH}_{2}$ & $1.30\left(-^{b)}\right)$ & 32.9 & $\mathrm{CH}_{2}$ & $1.50\left(-^{b)}\right)$ \\
\hline & & & & & & $1.28\left(-^{b)}\right)$ \\
\hline \multirow[t]{2}{*}{22} & 39.1 & $\mathrm{CH}_{2}$ & $0.95\left(-^{b)}\right)$ & 39.0 & $\mathrm{CH}_{2}$ & $0.94\left(\mathrm{dd}, 12.0,2.0, \mathrm{H}_{\mathrm{ax}}\right)$ \\
\hline & & & $1.47\left(-^{b)}\right)$ & & & $1.48\left(-,{ }^{b)} \mathrm{H}_{\mathrm{eq}}\right)$ \\
\hline 23 & 6.9 & $\mathrm{CH}_{3}$ & $0.94(\mathrm{~d}, 6.6)$ & 12.4 & $\mathrm{CH}_{3}$ & $1.01(\mathrm{~d}, 7.0)$ \\
\hline 24 & 14.5 & $\mathrm{CH}_{3}$ & $0.75(\mathrm{~s})$ & 16.6 & $\mathrm{CH}_{3}$ & $0.98(\mathrm{~s})$ \\
\hline 25 & 18.1 & $\mathrm{CH}_{3}$ & $0.91(\mathrm{~s})$ & 204.6 & $\mathrm{CH}$ & $10.20(\mathrm{~s})$ \\
\hline 26 & 19.1 & $\mathrm{CH}_{3}$ & $1.32(\mathrm{~s})$ & 19.6 & $\mathrm{CH}_{3}$ & $0.94(\mathrm{~s})$ \\
\hline 27 & 14.5 & $\mathrm{CH}_{3}$ & $0.99(\mathrm{~s})$ & 18.5 & $\mathrm{CH}_{3}$ & $0.96(\mathrm{~s})$ \\
\hline 28 & 30.4 & $\mathrm{CH}_{3}$ & $0.91(\mathrm{~s})$ & 31.9 & $\mathrm{CH}_{3}$ & $1.19(\mathrm{~s})$ \\
\hline 29 & 35.3 & $\mathrm{CH}_{3}$ & $1.27(\mathrm{~s})$ & 35.1 & $\mathrm{CH}_{3}$ & $0.95(\mathrm{~s})$ \\
\hline 30 & 32.4 & $\mathrm{CH}_{3}$ & $1.14(\mathrm{~s})$ & 31.6 & $\mathrm{CH}_{3}$ & $1.06(\mathrm{~s})$ \\
\hline
\end{tabular}

a) Assignments were confirmed by DEPT-135, HSQC, HMBC, ${ }^{1} \mathrm{H}-{ }^{1} \mathrm{H}$ COSY and NOESY experiments. b) Overlapping signals.
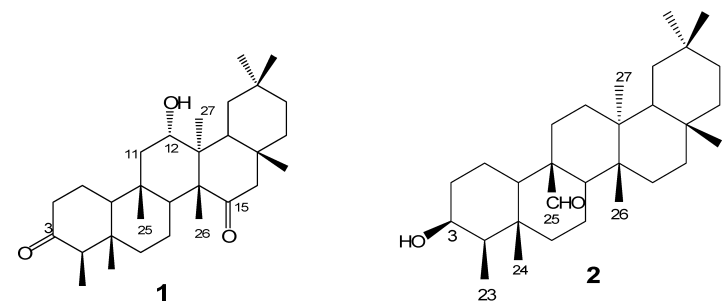

Fig. 1. Structures of Compounds $\mathbf{1}$ and $\mathbf{2}$

HSQC, HMBC and DEPT spectra suggested a friedelanetype triterpene skeleton $\left(\delta_{\mathrm{C}} 12.4, \mathrm{C}-23\right)$ having one oxymethine $\left(\delta_{\mathrm{C}} 71.7\right.$ and $\left.\delta_{\mathrm{H}} 3.80\right)$ and one aldehyde group $\left(\delta_{\mathrm{C}}\right.$ 204.6 and $\delta_{\mathrm{H}}$ 10.20). From the GC-MS, the fragment ion at $\mathrm{m} / \mathrm{z} 205$ confirmed the friedelane-type triterpene with the absence of any oxygen function on rings D and E. Also, the fragments at $m / z 125$ and 315 resulting from the cleavage of ring $\mathrm{B}$ suggested the location of one oxygen function on ring A. The HMBC spectrum showed correlations between the proton signal at $\delta_{\mathrm{H}} 3.80(\mathrm{H}-3)$ and carbon signals at $\delta_{\mathrm{C}} 49.5$ (C-4), 38.3 (C-5) and 12.4 (C-23), and between the aldehyde proton signal at $\delta_{\mathrm{H}} 10.20$ and the carbon signals $\delta_{\mathrm{C}} 52.5(\mathrm{C}$ 8), 51.6 (C-9), 59.6 (C-10) and 28.3 (C-11). Thus, the position of the hydroxy group was deduced to be at the $\mathrm{C}-3$ and the aldehyde function at C-25. The NOESY spectrum of $\mathbf{2}$ (Fig. 2) showed interactions between the aldehyde proton $\left(\delta_{\mathrm{H}}\right.$ $10.20)$ and the methyls $\mathrm{CH}_{3}-24\left(\delta_{\mathrm{H}} 0.98\right), \mathrm{CH}_{3}-26\left(\delta_{\mathrm{H}} 0.94\right)$ and proton $\mathrm{H}-18\left(\delta_{\mathrm{H}} 1.57\right)$. Furthermore, the stereochemistry of the carbon $\mathrm{C}-3$ was deduced to have $3 \beta-\mathrm{OH}$, based on the NOESY cross-peak from $\mathrm{H}-3\left(\delta_{\mathrm{H}} 3.80\right)$ to $\mathrm{CH}_{3}-23\left(\delta_{\mathrm{H}} 1.01\right)$; in addition, the coupling constant for $\mathrm{H}-3$ (q-like, $J=2.0 \mathrm{~Hz}$ ) confirmed its $\alpha$-equatorial orientation and consequently, the $\beta$-axial orientation of the hydroxy group in agreement to reported values. ${ }^{11)}$ Therefore, the structure of compound 2 was established as, 3 $\beta$-hydroxyfriedelan-25-al (Fig. 1). Its enantiomer with the $3 \alpha-\mathrm{OH}$ was isolated from Drypetes inaequalis. $^{8)}$

A number of studies have reported on the isolation of 
Table 2. Antimicrobial Activities of 1, 2, 3, 4, 5 and 7 (Each Conc. $200 \mu \mathrm{g} / \mathrm{ml}$ in DMSO)

\begin{tabular}{|c|c|c|c|c|c|c|c|}
\hline \multirow[b]{2}{*}{ Micro-organisms used } & \multicolumn{7}{|c|}{ Inhibition zone diameter in $\mathrm{mm}$} \\
\hline & 1 & 2 & 3 & 4 & 5 & 7 & $\begin{array}{c}\text { Gentamicin } \\
\text { (Control) }\end{array}$ \\
\hline Escherichia coli Gram-(-) & & & & & & 18 & 35 \\
\hline Salmonella typhi Gram-(-) & & & & & & 17 & 42 \\
\hline Shigella dysenteriae Gram-(-) & & & & & & & 30 \\
\hline Klebsiella pneumoniae Gram-(-) & & & & & & & 40 \\
\hline Pseudomonas aeruginosa $\operatorname{Gram}-(-)$ & & & & & & & 43 \\
\hline Staphylococcus aureus Gram-(+) & 17 & 14 & 11 & 10 & 15 & 21 & 34 \\
\hline
\end{tabular}
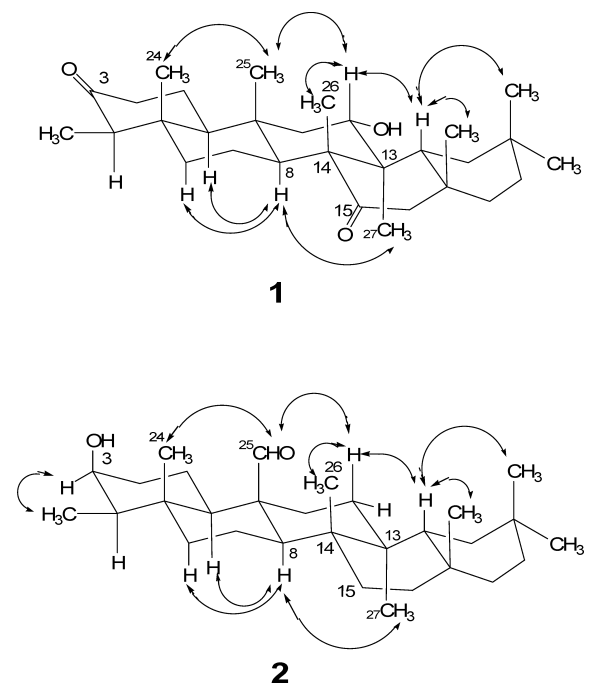

Fig. 2. Key NOESY Correlations Observed in Compounds $\mathbf{1}$ and $\mathbf{2}$

friedelane-type triterpenoids from plants and some have demonstrated diverse biological properties including antihuman immunodeficiency virus-1 (HIV-1) activity, ${ }^{12}$ ) antineoplastic/cytotoxic activity, ${ }^{13)}$ antitumor, antifungal, antiparasitic, antiviviral, anti-inflammatory and antimicrobial. ${ }^{14)}$

From the antimicrobial test results (Table 2), it appears that all the tested compounds, five friedelane derivatives 1 5 and the olean-12-ene saponin 7 exhibit an antimicrobial activity against Staphylococcus aureus. Compound 7 also reveals an antimicrobial activity against Gram-(-) Escherichia coli and Salmonella typhi. The activities of the six compounds $\mathbf{1}-\mathbf{5}$ and $\mathbf{7}$ were lower in comparison to that of gentamicin which was used as control molecule. The five friedelane derivatives $\mathbf{1}-\mathbf{5}$ showed no inhibitory activity on the five Gram-negative bacteria. It has been reported that, in general, Gram-positive bacteria are more sensitive to terpenes than Gram-negative and promising results were obtained against the Gram-positive bacteria Staphylococcus aureus. ${ }^{14)}$

\section{Experimental}

General The MPs were determined using a Kofler microhot stage apparatus. Optical rotations were measured on a Perkin-Elmer 241 polarimeter. IR spectra $\left(v_{\max }\right.$ in $\left.\mathrm{cm}^{-1}\right)$ were obtained from potassium pellets on a Nicolet 510 FT instrument. Mass spectra were recorded on a Micromass Q-TOF instrument, on a Nermag R10-10C spectrometer and a HP-5973 Mass Selective Detector. ${ }^{1} \mathrm{H}-\mathrm{NMR}(\delta[\mathrm{ppm}], J[\mathrm{~Hz}])$ and ${ }^{13} \mathrm{C}-\mathrm{NMR}$ spectra were recorded at $400 \mathrm{MHz}$ and $100 \mathrm{MHz}$, respectively, using a Bruker AC 400 spectrometer and a Varian Gemini $400 \mathrm{MHz}$ instrument. Multi-impulsional 1D and 2D NMR experiments (DEPT, ${ }^{1} \mathrm{H}-{ }^{1} \mathrm{H}$ COSY, ${ }^{1} \mathrm{H}-{ }^{1} \mathrm{H}$ NOESY, ${ }^{13} \mathrm{C}-{ }^{1} \mathrm{H}$ HSQC, ${ }^{13} \mathrm{C}-{ }^{1} \mathrm{H}$ HMBC) were performed using standard Bruker or
Varian Gemini micro-programs. Silica gel 60 (70-230 mesh) was used for column chromatography at normal pressure while silica gel $60 \mathrm{H}(5-$ $40 \mu \mathrm{m})$ and $60 \mathrm{AC}(20-40 \mu \mathrm{m})$ were used for column chromatography under compressed air (300 mbar). Precoated silica gel $60 \mathrm{~F}_{254}$ aluminium plates were used for thin layer chromatography and eluted with mixtures of solvents such as hexane/ $\mathrm{CH}_{2} \mathrm{Cl}_{2}(9: 1) ; \mathrm{CH}_{2} \mathrm{Cl}_{2} / \mathrm{MeOH}$ (19:1); $\mathrm{CH}_{2} \mathrm{Cl}_{2}$ $\mathrm{MeOH}(9: 1)$.

Plant Material The whole stems of Drypetes paxii Нuтch. (Euphorbiaceae) were collected by Mr. Victor Nana from East Province of Cameroon in October 2003. The herbarium specimen documenting the collection has been deposited in the National Herbarium, Yaounde, Cameroon.

Extraction and Isolation The whole stems of D. paxii were sun-dried, ground into a powder form $(10.0 \mathrm{~kg})$ which was macerated at room temperature with $\mathrm{MeOH}(3 \times 251)$ for $10 \mathrm{~d}$. The solvent was evaporated under reduced pressure to yield the total crude extract $(120.0 \mathrm{~g})$ which was subjected to CC over silica gel [60 (240- $400 \mathrm{mesh}), 800 \mathrm{~g}$ ]. A total of 208 fractions $(400 \mathrm{ml}$ each) were eluted with hexane, EtOAc and $\mathrm{MeOH}$ in increasing polarity. TLC permitted the combination of the resulting fractions into 8 groups of fractions coded A, B, C, D, E, F, G and $\mathrm{H}$ obtained as follows: A $(35 \mathrm{~g})$ [Fr. 1-25 (hexane-EtOAc $95: 5$ to $90: 10)$ ]; B (20 g) [Fr. 26-57 (hexane-EtOAc $85: 15$ to $70: 30)$ ]; C (16g) [Fr. 58-80 (hexane-EtOAc $65: 35$ to $60: 40)$ ]; D $(12 \mathrm{~g})$ [Fr. $81-107$ (hexane-EtOAc $55: 45$ to $40: 60)$ ]; E (10 g) [Fr. 108-130 (hexane-EtOAc 35:75 to 30:70)]; F (8g) [Fr. 131-170 (hexane-EtOAc $25: 75$ to 20:80)]; G (8g) [Fr. 171-192 (EtOAc-MeOH $100: 0$ to $95: 5)]$ and $\mathrm{H}(6 \mathrm{~g})$ [Fr. 193-208 (EtOAc-MeOH $90: 10$ to $85: 15)]$. Further CC over silica gel $60 \mathrm{C}(20-40 \mu \mathrm{m})$ of group A fractions using hexane-EtOAc $(95: 5)$ yielded compounds 2 (10 mg), 3 $(40 \mathrm{mg}), 4(20 \mathrm{mg})$ and $5(50 \mathrm{mg})$. Further CC over silica gel $60 \mathrm{C}(20-$ $40 \mu \mathrm{m})$ of group B fractions using hexane-EtOAc $(90: 10)$ afforded compounds $1(25 \mathrm{mg})$ and $6(70 \mathrm{mg})$. Further CC over silica gel $60 \mathrm{H}(5-$ $40 \mu \mathrm{m})$ of group $\mathrm{G}$ fractions by using EtOAc $(100 \%)$ yielded compounds 7 $(20 \mathrm{mg})$ and $8(80 \mathrm{mg})$.

$12 \alpha$-Hydroxyfriedelane-3,15-dione (1): Colourless amorphous crystals; mp 199-201 ${ }^{\circ} \mathrm{C}$ (hexane/EtOAc); TLC $R f: 0.8\left(\mathrm{CH}_{2} \mathrm{Cl}_{2} / \mathrm{MeOH}\right.$; 19 : 1); violet spot developed on spraying with aqueous $\mathrm{H}_{2} \mathrm{SO}_{4}(50 \%)$. $[\alpha]_{\mathrm{D}}^{20}+24.6^{\circ}$ $\left(c=0.70, \mathrm{CHCl}_{3}\right) ;{ }^{1} \mathrm{H}$ - and ${ }^{13} \mathrm{C}-\mathrm{NMR}$ spectral data $\left(400,100 \mathrm{MHz}, \mathrm{CDCl}_{3}\right)$, see Table 1. IR $(\mathrm{KBr}) \mathrm{cm}^{-1}: 3410(\mathrm{OH}), 3015,1725(\mathrm{C}=\mathrm{O}), 1260,1180$, 892; $\mathrm{Cl} / \mathrm{NH}_{3}$ MS $m / z: 457[\mathrm{M}+\mathrm{H}]^{+}, 474\left[\mathrm{M}+\mathrm{NH}_{4}\right]^{+}$. EI-MS $m / z: 456$ $[\mathrm{M}]^{+}, 438\left[\mathrm{M}-\mathrm{H}_{2} \mathrm{O}\right]^{+}, 262,219,192,177 ; \mathrm{HR}^{2} \mathrm{TOF} \mathrm{ES}^{+} \mathrm{MS} m / z: 456.7152$ (Calcd for $\mathrm{C}_{30} \mathrm{H}_{48} \mathrm{O}_{3} 456.7145$ ).

$3 \beta$-Hydroxyfriedelan-25-al (2): Colourless amorphous powder; mp 160$162^{\circ} \mathrm{C}$ (hexane/EtOAc); TLC Rf: $0.9\left(\mathrm{CH}_{2} \mathrm{Cl}_{2}\right) ;[\alpha]_{\mathrm{D}}^{20}-14.6^{\circ} \quad(c=0.70$, $\left.\mathrm{CHCl}_{3}\right) ;{ }^{1} \mathrm{H}$ - and ${ }^{13} \mathrm{C}-\mathrm{NMR}$ spectral data $\left(400,100 \mathrm{MHz}, \mathrm{CDCl}_{3}\right)$, see Table 1. IR (KBr) cm ${ }^{-1}: 3395(\mathrm{OH}), 3029,1722(\mathrm{C}=\mathrm{O}), 1260,1178,890$;. GCMS $m / z: 442[\mathrm{M}]^{+}, 315,205,125 . \mathrm{CI} / \mathrm{NH}_{3} \mathrm{MS} m / z: 443[\mathrm{M}+\mathrm{H}]^{+}, 460$ $\left[\mathrm{M}+\mathrm{NH}_{4}\right]^{+}$; HR TOF ES ${ }^{+}$MS m/z: 442.3825 (Calcd for $\mathrm{C}_{30} \mathrm{H}_{50} \mathrm{O}_{2}$ 442.3811).

Antimicrobial Activity. Microbial Strains A total of 6 selected pathogenic bacteria belonging to five Gram- $(-)$ (Escherichia coli, Salmonella typhi, Shigella dysenteriae, Klebsiella pneumoniae and Pseudomonas aeruginosa) and one Gram-(+) (Staphylococcus aureus) were clinically isolated from patients at the "Centre Pasteur de Yaoundé" Cameroon. They were maintained on agar slant at $4{ }^{\circ} \mathrm{C}$ in the Laboratory of the Applied Microbiology and Molecular Pharmacology (Faculty of Science, Yaounde).

Antimicrobial Assays Antimicrobial activity was evaluated using the agar diffusion method, according to the NCCLS (2002) protocol ${ }^{15)}$ with slight modifications. Briefly, sterile cylinders of $6 \mathrm{~mm}$ were used to make wells inside Mueller-Hinton agar plates. The plates were inoculated with 
$0.2 \mathrm{ml}$ of the test micro-organisms equivalent to $5 \times 10^{5}$ colony forming unit $(\mathrm{CFU}) / \mathrm{ml}$. All the compounds and the reference antibiotic gentamycin were dissolved in dimethyl sulfoxide (DMSO) or in heated sterilized distilled water at a concentration $200 \mu \mathrm{g} / \mathrm{ml}$. Wells were filled with $0.15 \mathrm{ml}$ of solution of each test compound, the positive control drug (gentamicin) and the negative control DMSO, and allowed to diffuse for $45 \mathrm{~min}$ at $4{ }^{\circ} \mathrm{C}$. The plates were incubated at $37^{\circ} \mathrm{C}$ for $24 \mathrm{~h}$. The sensitivity was recorded by measuring the clear zone of growth inhibition around the wells ( $\mathrm{mm}$ diameter). Each set was tested in triplicate.

Acknowledgements One of the authors (J. Wandji) is grateful for grant $\left(\mathrm{N}^{\circ} \mathrm{F} / 2624-3 \mathrm{~F}\right)$ from the International Foundation for Science (Sweden), and to the sponsorship of the "Université Paris Descartes, France" during his multiple research visits to Paris

\section{References}

1) Bouquet A., Debray L., "Plantes Médicinales de la Côte-d'Ivoire," Travaux et documents de l'ORSTOM N³2, 1974, pp. 82-87.

2) Dalziel J. M., "The Useful Plants of West Tropical Africa," The Crown Agents for the Colonies, London, 1937, pp. 140-141.

3) Irvine R. F., "Woody Plants of Ghana," Oxford University Press, London, 1961, pp. 223-226.

4) Walker A. R., Sillans R., Trochain J. L., "Les plantes utiles du Gabon," ed. by Lechevalier P., 12-Rue de Tournon Paris VI, 1961, pp. 165166.
5) Chungag-Anye N. B., Njamen D., Dongmo A. B., Wandji J., Nguelefack T. B., Wansi J. D., Kamanyi A., Fomum Z. T., Pharmacol. Lett., 11, 61-63 (2001).

6) Chungag-Anye N. B., Njamen D., Dongmo A. B., Wandji J., Fomum Z. T., Nguelefack T. B., Kamanyi A., Pharm. Biol., 41, 26-30 (2002).

7) Wansi J. D., Wandji J., Lallemand M. C., Chiozem D. D., Iqbal M. C., Tillequin F., Fomum T. Z., BLACPMA, 6, 5-10 (2007).

8) Awanchiri S. S., Dufat H. T. V., Shirri C. J., Dongfack J. M. D., Nguenang G. M., Boutefnouchet S., Fomum Z. T., Seguin E., Verite P., Tillequin F., Wandji J., Phytochemistry, 70, 419-423 (2009).

9) Mahato S. B., Kundu A. P., Phytochemistry, 37, 1517-1575 (1994).

10) Wandji J., Tillequin F., Mulholland A. D., Temgoua D. A., Wansi J. D., Seguin E., Fomum Z. T., Phytochemistry, 63, 453- 456 (2003).

11) Salazar G. C., Silva G. D. F., Duarte L. P., Vieira Filho S. A., Lula I. S., Magn. Reson. Chem., 38, 977-980 (2000).

12) Chen S., Yoshiki K., Susan L. M. N., Kuo H. L., Curr. Top. Med. Chem., 3, 155-169 (2003).

13) Setzer W. N., Setzer M. N., Mini Rev. Med. Chem., 3, 540-556 (2003).

14) Paduch R., Kandefer-Szerszeń M., Trytek M., Fiedurek J., Arch. Immunol. Ther. Exp., 55, 315-327 (2007).

15) NCCLS National Committee for Clinical Laboratory Standards, "Methods for Dilution Antimicrobial Susceptibility Test for Bacteria That Grow Aerobically," 3rd ed., Approved standard NCCLS document M100-812, NCCLS, Wayne, PA, 2002. 